\title{
Respuesta a la suplementación con propilenglicol en vacas multiovuladas, para la producción de embriones ${ }^{1}$
}

\section{Propylene glycol supplementation response in multiovulated cows for embryo production}

\author{
Roger Molina-Coto ${ }^{2}$, Carlos Arroyo-Oquendo², Daniel Carballo-Guerrero ${ }^{3}$, Jorge Alberto Elizondo-Salazar ${ }^{4}$
}

\begin{abstract}
Resumen
La producción de embriones in vivo es una herramienta para la mejora genética bovina. Los protocolos de sincronización-multiovulación de las hembras donantes de embriones, así como la facilidad de la colecta de embriones han evolucionado, sin embargo, aún se debe maximizar la técnica. El objetivo de este trabajo fue evaluar el efecto de la administración de propilenglicol (PPG) en vacas donantes multiovuladas sobre la producción y calidad de embriones. El estudio se llevó a cabo en el cantón de Pococí, Limón, Costa Rica, desde febrero del año 2011 a enero del 2012. Se suministraron $300 \mathrm{ml}$ diarios de PPG por vía oral, desde una semana previa y hasta una semana posterior al inicio del protocolo de sincronización-multiovulación de la vaca donante. Las colectas de embriones se realizaron cada dos meses y se utilizaron siete vacas. Se realizaron evaluaciones ováricas por medio de ultrasonografía antes y durante los días del tratamiento de sincronización-multiovulación. Se colectaron y clasificaron los embriones con una edad de siete días. El tratamiento con PPG no afectó el número y tamaño de los folículos ováricos medidos por ultrasonografía. De igual manera, la cantidad de estructuras colectadas y su calidad no mejoraron con la suplementación. Los resultados del experimento sugieren que otros factores, ajenos al efecto de la suplementación con PPG, afectan la respuesta animal ante los tratamientos para multiovulación y colecta de embriones.
\end{abstract}

Palabras clave: embriones, ganado bovino, ovulación inducida, folículos ováricos, reproducción.

\begin{abstract}
Multiovulation and embryo transfer is a technique used in genetic breeding programs. The synchronizationmultiovulation protocols of bovine embryo donors, as well as the embryo collection technique have improved, however, the technique should still be maximized. This study was done with the objective of evaluating the effect of the administration of propylene glycol (PPG) in multiovulated donor cows on the production and quality of embryos. The study was conducted in Pococi, Limon, Costa Rica, from February 2011 to January 2012. Daily and oral
\end{abstract}

1 Recibido: 25 de octubre, 2017. Aceptado: 28 de febrero, 2018. Este trabajo formó parte del proyecto 737-B1-010 inscrito en la Vicerrectoría de Investigación de la Universidad de Costa Rica, San José, Costa Rica.

2 Universidad de Costa Rica, Facultad de Ciencias Agroalimentarias. Escuela de Zootecnia, San José, Costa Rica. roger.molina@ucr.ac.cr, carlos.arroyo@ucr.ac.cr

3 Asistencia Veterinaria de Costa Rica, Convenio de Cooperación con la Universidad de Costa Rica, San José, Costa Rica. cargueda@hotmail.com

4 Universidad de Costa Rica, Facultad de Ciencias Agroalimentarias. Estación Experimental Alfredo Volio Mata, Cartago, Costa Rica. jorge. elizondosalazar@ucr.ac.cr 
administration of $300 \mathrm{mg}$ PPG was given from one week prior to one week after the starting of the synchronizationmultiovulation protocol. Embryo flushing was performed every two months and seven cows were used. Ovarian evaluations were performed using transrectal ultrasonography before and during the days of the synchronizationmultiovulation treatment. The embryos were collected and classified at the age of seven days. Treatment with PPG did not affect the number and size of ovarian follicles as measured by ultrasonography. Likewise, the number of structures collected and their quality did not improve with supplementation. The results of the experiment suggest that other factors, unrelated to the effect of PPG supplementation, affect the animal response to treatments for multiovulation and embryo collection.

Keywords: embryos, cattle, induced ovulation, ovarian follicles, reproduction.

\section{Introducción}

La producción de embriones bovinos in vivo acelera la reproducción de hembras que expresan rasgos de interés productivo y por tanto, acelera el proceso de mejora genética. Existe una serie de tratamientos hormonales para multiovulación y la respuesta a estos es determinante para el éxito de la técnica de producción y transferencia de embriones in vivo (Palma, 2008), la cual ha progresado desde sus inicios para ser económicamente viable. Los protocolos de sincronización y multiovulación, así como la técnica de colección y conservación de embriones también han mejorado; sin embargo, en los últimos cuarenta años el promedio de embriones transferibles por colecta se ha mantenido constante (siete para ganado de carne y 6,3 para ganado de leche) (Hasler, 2014). Factores como la lactancia, la estación del año, el intervalo entre colectas, la vaca donante y el evaluador de los embriones en la colecta, son algunos de los factores que afectan los resultados de la producción de embriones (Callesen et al., 1995; Chebel et al., 2008).

En general, el estatus nutricional de las hembras bovinas influye sobre la fertilidad de las mismas (Butler, 2014). En el bovino, la fuente energética primordial son los ácidos grasos volátiles que se producen en el rumen (Dijkstra, 1994). Los ovarios, lugar donde se desarrollan las diferentes estructuras relacionadas con la respuesta a los tratamientos hormonales para multiovulación, son dependientes de la glucosa y fuente energética (Santos, 2008), y requieren de glucosa para llevar a cabo el ciclo ovárico normal o responder a las alteraciones exógenas intencionales.

En busca de mejorar el estatus nutricional de los rumiantes y aportar la energía necesaria para suplir los requerimientos para producción y reproducción, se han utilizado diversos suplementos. El propilenglicol (PPG), también conocido por los nombres de 1,2-propanodiol, metiletilen glicol, metil glicol y monopropilenglicol, es un alcohol de uso industrial que se ha utilizado para la suplementación de hembras bovinas (Christensen et al., 1997; Hoedemaker et al., 2004; Chagas et al., 2007; Moallem et al., 2007). El PPG se caracteriza por ser un producto de alta estabilidad y que no se considera peligroso (ATSDR, 1997); además, se ha usado como anticongelante para la fabricación de cerveza, solvente de productos farmacéuticos, emulsificante en comidas, desinfectante de aire e inhibidor de la fermentación y crecimiento de mohos (Windholz et al., 1983).

En el animal rumiante, el PPG es convertido a propionato en el rumen y este a piruvato y glucosa en el hígado (Jenkins et al., 2015). Algunos factores metabólicos responden a las concentraciones de glucosa, entre estos la insulina, la cual es una señal metabólica para la activación de factores de crecimiento y por tanto, influye sobre el crecimiento de estructuras ováricas como los folículos y el cuerpo lúteo (Laskowski et al., 2016).

La manera en la que se administre el PPG es determinante en sus efectos, se usa dentro de la ración, mezclado con el concentrado, de manera oral directa, vía fístula ruminal o por medio de una sonda esofágica (Christensen et al., 1997; Chung et al., 2009). La vía oral directa aumentó las concentraciones de ácido propiónico en rumen 
de manera más rápida que las otras formas de administración; mientras que, las concentraciones de ácidos grasos no esterificados (NEFA) disminuyeron por la misma vía (Christensen et al., 1997). Esto convierte al PPG en una herramienta para tratar las enfermedades metabólicas post parto como la cetosis (Piantoni y Allen, 2015). El PPG aumenta los niveles séricos de factor de crecimiento similar a la insulina (IGF1) y disminuye los NEFAs y betahidroxibutirato (BHBA), esta mejora metabólica favorece el crecimiento de tejido como los folículos (Hoedemaker et al., 2004). Pese a la obtención de estos resultados positivos, los mismos autores recomiendan investigar aspectos económicos, de producción de leche, salud animal y fertilidad en torno a la suplementación con PPG.

Debido a la necesidad de buscar alternativas que mejoren la producción de embriones por medio de la multiovulación y a las características de un suplemento como el PPG, se realizó esta investigación con el objetivo de evaluar el efecto de la administración de propilenglicol (PPG) en vacas donantes multiovuladas sobre la producción y calidad de embriones.

\section{Materiales y métodos}

A nivel de campo, la investigación se realizó desde el 10 de febrero del año 2011 al 27 de enero del año 2012 en Guápiles, Pococí, en la Unidad de Reproducción Animal (URA) de la Escuela de Zootecnia de la Universidad de Costa Rica, bajo el convenio de cooperación, entre el Ministerio de Agricultura y Ganadería (MAG), el Instituto Nacional de Innovación y Transferencia en Tecnología Agropecuaria (INTA) y la Universidad de Costa Rica (UCR). La finca se encuentra a una altitud de $249 \mathrm{msnm}$. La precipitación y temperaturas tenidas durante las 62 semanas del periodo experimental se presentan en las Figuras 1 y 2 (datos de la sub-estación meteorológica de la Corporación Bananera Nacional (CORBANA), en la Rita de Guápiles, Pococí).

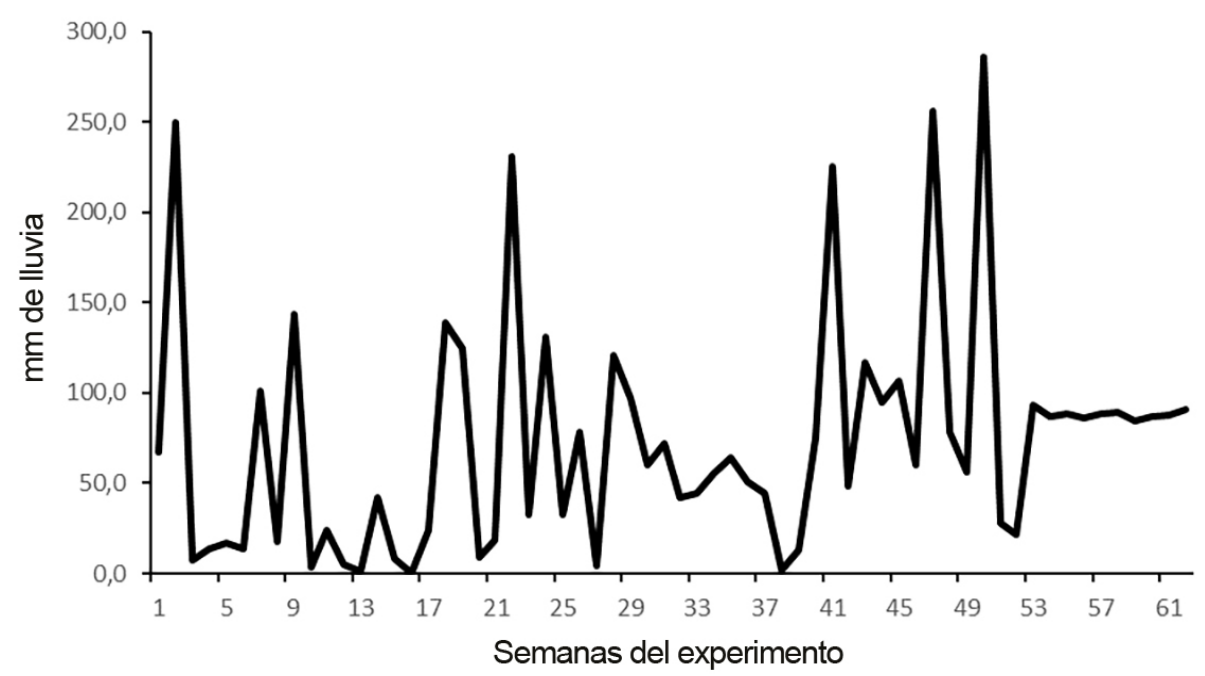

Figura 1. Precipitación promedio semanal registradas desde enero 2011 a febrero 2012 en Guápiles, Pococí, Costa Rica. Figure 1. Mean weekly precipitation recorded from January 2011 to February 2012 in Guapiles, Pococi, Costa Rica. 


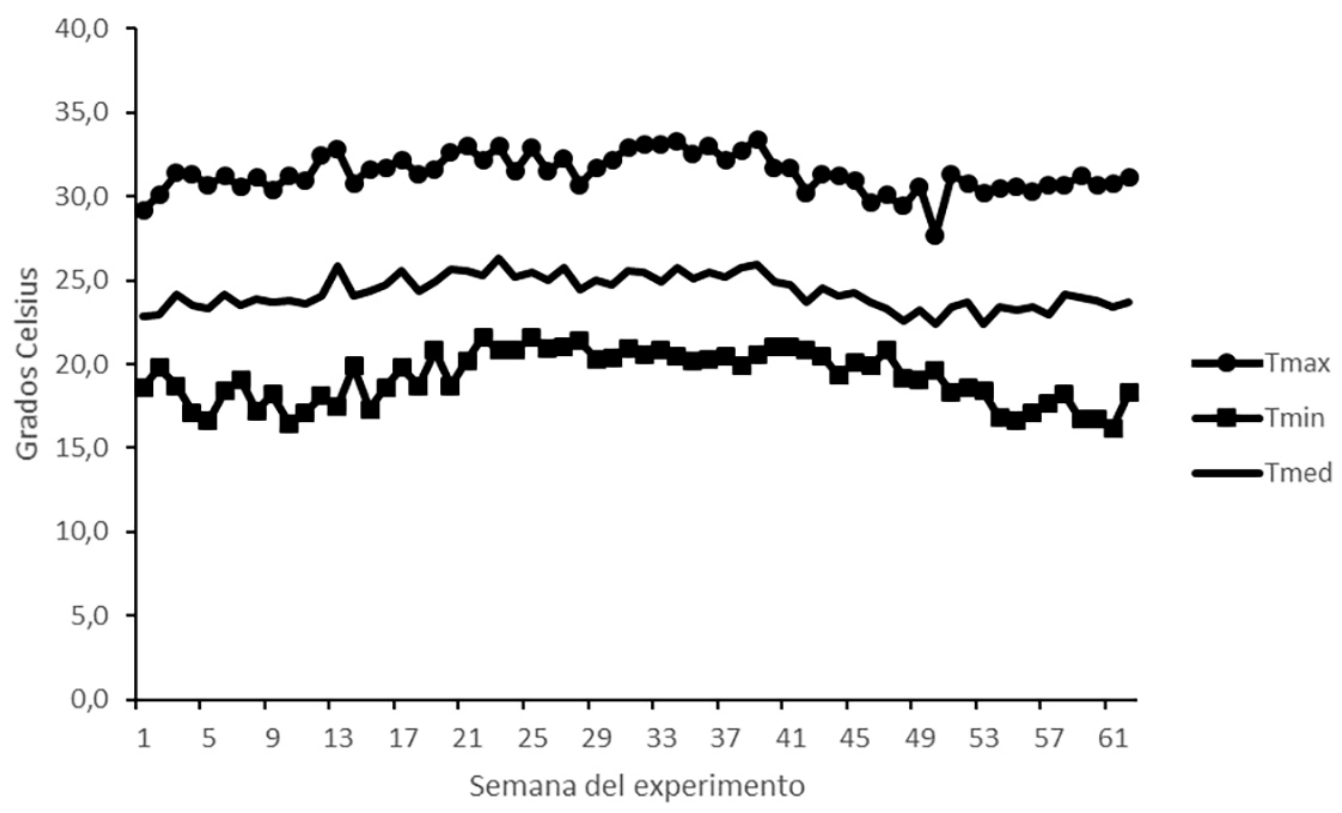

Figura 2. Promedio semanal de temperaturas máxima (Tmax), mínima (Tmin) y media (Tmed) desde enero 2011 a febrero 2012 en Guápiles, Pococí, Costa Rica.

Figure 2. Average weekly maximun (Tmax), minimun (Tmin) and mean (Tmed) temperatures from January 2011 to February 2012 in Guapiles, Pococi, Costa Rica.

Los procedimientos de este proyecto fueron aprobados por el Comité Institucional de Cuido y Uso de Animales (CICUA), de la Universidad de Costa Rica.

\section{Animales}

Se seleccionaron siete hembras donantes con desempeño conocido en la producción de embriones por medio de multiovulación. Dos hembras de la raza Simbrah, dos Canchim (5/8 Charolais x 3/8 Nelore), dos Limousine y una Nelore. El promedio de peso de las hembras durante el experimento fue $529 \pm 72 \mathrm{~kg}$ y mantuvieron una condición corporal promedio de 3,75 $\pm 0,5$ (promedio \pm desviación estándar) (escala de 1 a 5, siendo 1 el animal con menos reservas corporales y 5 el animal con más reservas). Ninguna de las hembras estaba en lactancia o con ternero al pie. Las hembras fueron inseminadas con semen congelado proveniente de toros de fertilidad comprobada. Los animales se mantuvieron en pastoreo rotacional en pasturas nativas y recibían un suplemento diario que consistió en cáscara de banano a libre consumo y una mezcla líquida de urea-melaza (aproximadamente $100 \mathrm{~g}$ de urea y 0,5 kg de melaza por animal por día).

\section{Diseño del experimento}

Se realizaron seis colectas de embriones (seis repeticiones) en las siete hembras, con un intervalo entre colectas de al menos cuarenta y ocho días. El tratamiento aplicado consistió en una infusión oral de $300 \mathrm{ml}$ de PPG (pureza $>99,8 \%$ ) el cual se suministró durante quince días, siete antes (-7) de iniciar, el día 0 (inicio de protocolo de sincronización de celo y multiovulación) y hasta el día 7 de tratamiento (día de finalización de aplicación de la 
hormona superestimulante, FSH). Hubo al menos 105 días entre las colectas de los animales que les correspondía el tratamiento con PPG. La forma de administración del PPG fue oral, mediante una jeringa de $300 \mathrm{ml}$ con una cánula de $25 \mathrm{~cm}$. La línea de tiempo del diseño del experimento de la suplementación con PPG y los días de evaluación se observan en la Figura 3. Durante los días de evaluación con ultrasonido (US) se observaron los ovarios de las vacas.

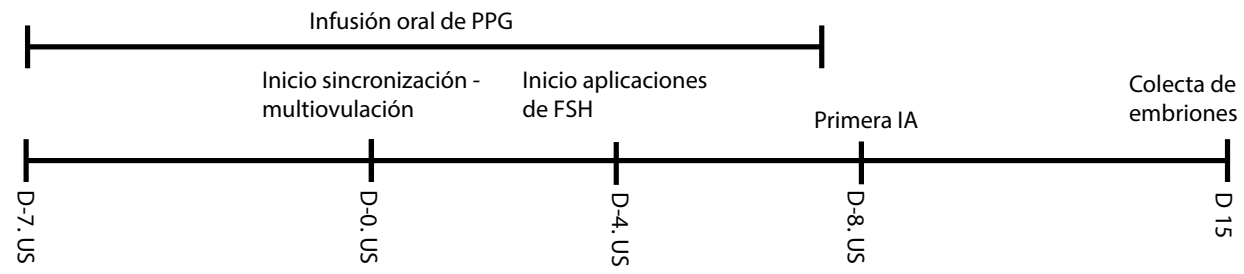

Figura 3. Línea de tiempo del experimento del tratamiento de suplementación con propilenglicol (PPG) en vacas, razas Simbrah, Canchim (5/8 Charolais x 3/8 Nelore), Limousine y Nelore. Guápiles, Pococí, Costa Rica. Enero de 2011 a febrero de 2012. $\mathrm{PPG}=$ propilenglicol, $\mathrm{FSH}=$ hormona folículo estimulante, $\mathrm{D}=$ día, $\mathrm{US}=$ evaluación con ultrasonido, IA: inseminación artificial.

Figure 3. Timeline of the propylene glycol (PPG) supplementation treatment in cows Simbrah, Canchim (5/8 Charolais $\mathrm{x} 3 / 8$ Nellore), Limousine and Nellore breeds. Guapiles, Pococi, Costa Rica. January 2011 to February 2012. $\mathrm{PPG}=$ propylene glycol, $\mathrm{FSH}=$ follicle stimulating hormone, $\mathrm{D}=$ day, $\mathrm{US}=$ ultrasound evaluation, $\mathrm{IA}=$ artificial insemination .

Los animales se distribuyeron al azar para la primera repetición del experimento entre los que recibieron PPG, y los que no (control). Tres animales iniciaron la primera repetición con PPG, mientras que, cuatro no lo recibieron, para la segunda repetición, se alternó, de manera que los tres que fueron tratados con PPG en la primera repetición se convirtieron en los animales control (su propio control) en la segunda repetición y viceversa. Esta rotación se volvió a realizar para las repeticiones subsecuentes hasta que cada animal, de manera alterna, tuviera tres colectas en las que fue tratado con PPG y tres como control, para un total de seis colectas por vaca (repeticiones).

Todos los tratamientos fueron aplicados por personal entrenado de la Unidad de Reproducción Animal de la Finca Experimental. El protocolo de sincronización y multiovulación utilizado incluyó la inserción de un dispositivo intravaginal liberador de progesterona ( $1 \mathrm{~g}$ de progesterona), una inyección con 2,5 $\mathrm{mg}$ de benzoato de estradiol y 62,5 mg de progesterona inyectada en el día cero. Del día cuatro al siete, se aplicó la hormona folículo estimulante (extracto comercial de FSH porcina) en dosis decrecientes cada 12 h. La dosis de FSH, se estableció para cada animal, con base en el historial de multiovulación al cual había sido sometido antes de este experimento. El animal en que menos dosis de FSH se utilizó fue de $200 \mathrm{mg}$, mientras que el de máxima dosis fue de $400 \mathrm{mg}$. En el día seis se inyectó $0,15 \mathrm{mg}$ de un análogo de prostaglandina (cloprostenol dextrógiro) en la mañana y 0,15 $\mathrm{mg}$ en la tarde. El día siete se retiró el dispositivo intravaginal, en el día ocho por la mañana se aplicaron 0,25 mg de un análogo de la hormona liberadora de gonadotropinas (acetato de gonadorelina), y se realizó la inseminación artificial en la tarde del día ocho y en la mañana del día nueve. El día quince del tratamiento se realizó la colecta de embriones por vía transvaginal no quirúrgica.

\section{Toma y evaluación de datos}

Se evaluaron las estructuras ováricas de las hembras durante los días -7, 0, 4 y 8 del tratamiento, por medio de ultrasonido para uso animal equipado con una sonda lineal transrectal de 7,5 $\mathrm{MHz}$ de frecuencia. Cada ecografía ovárica fue grabada e identificada individualmente, por medio de un dispositivo MP4 conectado al ultrasonido, 
para ser posteriormente evaluado en una computadora. Se contabilizaron los cuerpos lúteos y folículos, además, los folículos se clasificaron según su diámetro en tres clases: Clase 1: $\leq 5 \mathrm{~mm}$, Clase 2: 6 a $9 \mathrm{~mm}$ y Clase 3: $\geq 10 \mathrm{~mm}$, con base en la escala de medición del ultrasonido.

El día de la colecta de embriones (día quince), se determinó el número de cuerpos lúteos en los ovarios por medio de palpación transrectal. La selección y clasificación de las estructuras colectadas (embriones y ovocitos) se realizó siguiendo el procedimiento indicado por Bó y Mapletoft (2013), que se apega a la clasificación de la Sociedad Internacional de Transferencia de Embriones (IETS, por sus siglas en inglés). Dicha clasificación consiste en cuatro categorías (1, 2, 3 y 4), donde la mejor calidad es 1 y disminuye conforme aumenta el número de categoría, bajo esta premisa, se consideraron embriones transferibles a los de calidad 1 y 2 . La evaluación de las estructuras colectadas se realizó en un estereomicroscopio de luz reflejada por espejo con un rango de aumentos de 6,7 a 45X. Tanto para las evaluaciones con ultrasonido, como los resultados de las colectas, se tomaron en hojas de campo que fueron digitalizadas en libros electrónicos para luego ser analizados con el software estadístico SAS 9.4.

\section{Análisis de datos}

Dos animales fueron retirados del experimento, uno en la repetición cuatro y otro en la repetición seis. Por lo tanto, la información referida a la colecta de los embriones de estos animales no se registró, únicamente se utilizó la información de las evaluaciones por ultrasonido cuando el animal estuvo disponible. La información de las ecografías se analizó por medio de un modelo ANOVA de mediciones repetidas, donde las variables de clasificación fueron el animal, tratamiento, repetición y día de evaluación. Se evaluaron las interacciones de segundo orden entre las variables. Las interacciones no significativas fueron removidas del modelo. Para el análisis de la información generada el día de la colecta de embriones (día quince del experimento), también se utilizó un modelo ANOVA de mediciones repetidas que incluyó las variables de clasificación de animal, tratamiento, repetición y toro. La matriz de varianzas utilizadas para los dos análisis fue la simétrica compuesta. Además, se realizó un análisis de correlación de Pearson entre las variables medidas con ultrasonido el día cero del experimento y las variables obtenidas el día de la colecta de embriones en el día quince del experimento. La significancia estadística se declaró con un valor $\mathrm{P}<0,05$ y las tendencias de $\mathrm{P}=0,05$ a $\mathrm{P}=0,09$.

\section{Resultados}

\section{Respuesta ovárica a la suplementación con PPG medida con ultrasonido}

La suplementación con PPG no afectó la actividad ovárica cuando se evaluó el número de folículos presentes por clase por medio de ultrasonografía ( $\mathrm{P}=0,53$ para folículos clase $3, \mathrm{P}=0,90$ para folículos clase 2 y $\mathrm{P}=0,81$ para folículos clase 1) (Figura 4). Para los folículos clase 3, se observó una interacción entre el tratamiento y la repetición ( $\mathrm{P}=0,025)$. Para las repeticiones 2 y 6 , la producción de folículos clase 3 fue mayor en el grupo sin PPG. Para la repetición 5, el tratamiento con PPG generó mayor cantidad de folículos clase 3, mientras que, en las demás repeticiones no se presentaron diferencias entre los tratamientos. Para los folículos clase 1 y 2 , no se presentó dicha interacción (tratamiento $\times$ repetición, P>0,05), sin embargo, la repetición sí afectó la cantidad de folículos para ambas clases (Clase 1, $\mathrm{P}=0,048$; Clase 2, $\mathrm{P}=0,016$ ).

El día del tratamiento en que se realizó la ecografía, afectó significativamente la población folicular de todas las clases $(\mathrm{P}<0,0001)$. Esto era esperado en respuesta al protocolo de sincronización y multiovulación utilizado. Los promedios de la cantidad de folículos por clase en cada día de evaluación se aprecian en la Figura 5. 


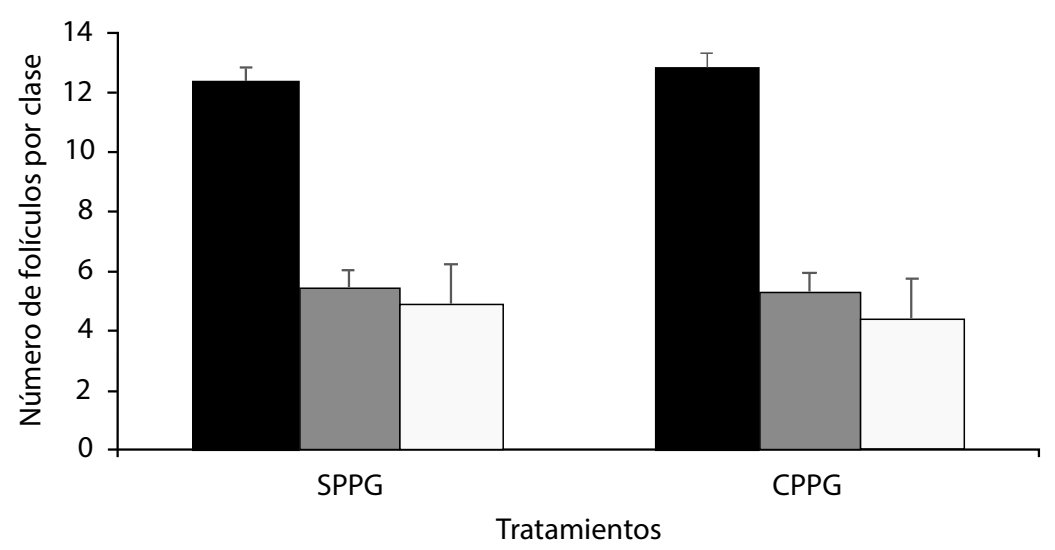

Clase $3 \square$ Clase $2 \square$ Clase 1

Figura 4. Número promedio de folículos por clase para cada tratamiento (con (CPPG) y sin (SPPG) suplementación con propilenglicol, durante los días -7, 0, 4 y 8 de evaluación con ultrasonido en vacas, razas Simbrah, Canchim (5/8 Charolais x 3/8 Nelore), Limousine y Nelore. Guápiles, Pococí, Costa Rica. Enero 2011 a febrero 2012.

$\mathrm{P}>0,1$ en cada clase.

$\mathrm{SPPG}=\sin$ suplemento de propilenglicol, $\mathrm{CPPG}=$ con suplemento de propilenglicol. Clase $1: \leq 5 \mathrm{~mm}$, Clase 2: 6 a $9 \mathrm{~mm}$ y Clase 3: $\geq 10 \mathrm{~mm}$, con base en la escala de medición del ultrasonido.

Figure 4. Average number of follicles per class for each treatment (with (CPPG) and without (SPPG) propylene glycol supplementation) during days -7, 0,4 and 8 of the ultrasound evaluation in cows Simbrah, Canchim (5/8 Charolais x 3/8 Nellore), Limousine and Nellore breeds. Guapiles, Pococi, Costa Rica. January 2011 to February 2012. $\mathrm{P}>0.1$ in each class.

$\mathrm{SPPG}=$ no supplement of propylene glycol; $\mathrm{CPPG}=$ with supplement of propylene glycol. Class $1: \leq 5 \mathrm{~mm}, \mathrm{Class} 2: 6$ to $9 \mathrm{~mm}$ and Class $3: \geq 10 \mathrm{~mm}$, based on the ultrasound machine caliper.

\section{Respuesta en estructuras colectadas a la suplementación con PPG}

La cantidad de embriones transferibles (calidad 1 y 2, según clasificación recomendada por Bó y Mapletoft (2013), se presenta en el Cuadro 1; se observa para los embriones transferibles la cantidad promedio absoluta por cada repetición del experimento, con sus valores máximos y mínimos. Además, se presenta el número de animales (n) en cada repetición para cada tratamiento.

El tratamiento con PPG generó resultados similares al control en cuanto a la evaluación de estructuras colectadas $(\mathrm{P}=0,48$ para el total de estructuras colectadas; $\mathrm{P}=0,85$ para el total de estructuras fertilizadas; $\mathrm{P}=0,94$ para el total de embriones clasificados como calidad $1 ; \mathrm{P}=0,49$ para el total de embriones calidad 1 en estado de mórula; $\mathrm{P}=0,36$ para el total de embriones calidad 1 en estado de blastocito; $\mathrm{P}>0,05$ para embriones clasificados como calidad 2, 3 y 4; $\mathrm{P}=0,35$ para el total de estructuras infertilizadas). En la Figura 6, se presenta la distribución en los tratamientos de las estructuras colectadas, se muestra además, el número de estructuras fertilizadas e infertilizadas. En la Figura 7, se desglosa el número de estructuras colectadas (embriones) de acuerdo con su calidad dentro de cada tratamiento. En el presente estudio se determinó que las calidades de los embriones fueron similares independientemente del tratamiento.

El efecto de la repetición no alteró $(\mathrm{P}>0,05)$ las variables del total de estructuras colectadas, cantidad de embriones fertilizados, embriones, mórulas calidad 1, blastocitos calidad 1, número de estructuras infertilizadas 0 embriones calidad 2 y 4 , únicamente afectó los embriones calidad 3 ( $\mathrm{P}=0,033)$. 


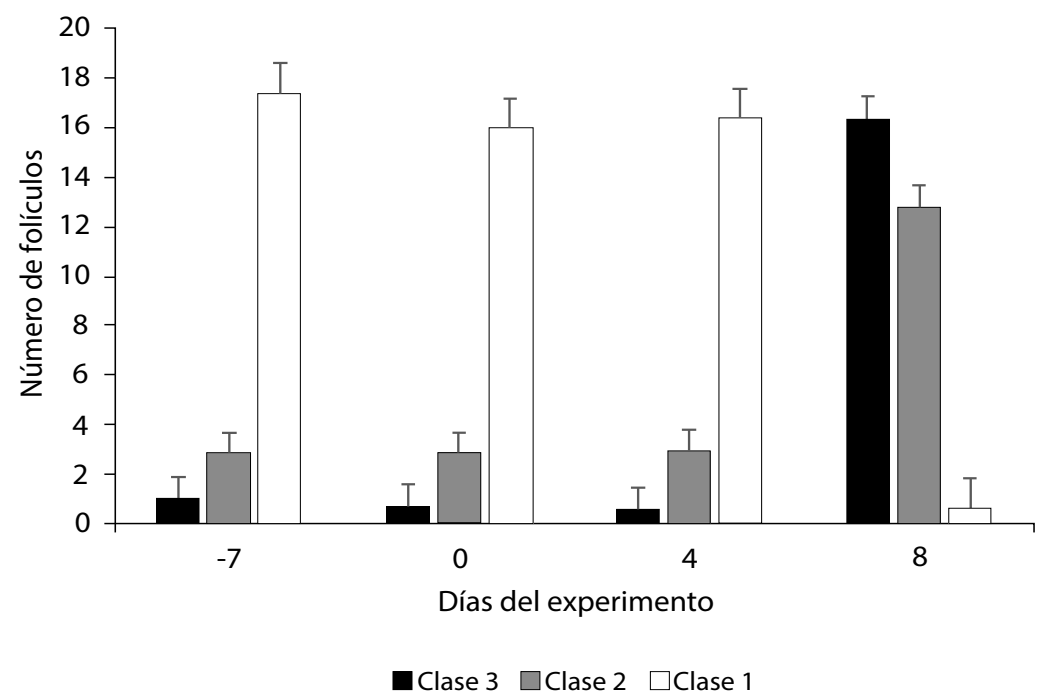

Figura 5. Número de folículos por clase durante los días de evaluación del experimento de suplementación con propilenglicol (PPG) en vacas razas Simbrah, Canchim (5/8 Charolais x 3/8 Nelore), Limousine y Nelore. Guápiles, Pococí, Costa Rica. Enero 2011 a febrero 2012.

Día $0=$ inicio del tratamiento de sincronización y multiovulación. $\mathrm{P}<0,0001$ para todas las clases de folículos. Clase 1: $\leq 5 \mathrm{~mm}$, Clase 2: 6 a $9 \mathrm{~mm}$ y Clase 3: $\geq 10 \mathrm{~mm}$, con base en la escala de medición del ultrasonido.

Figure 5. Number of follicles per class during the days of evaluation of the experiment of propylene glycol (PPG) supplementation in cows Simbrah, Canchim (5/8 Charolais x 3/8 Nellore), Limousine and Nellore breeds. Guapiles, Pococi, Costa Rica. January 2011 to February 2012.

Day $0=$ start of synchronization and multiovulation treatment. $\mathrm{P}<0.0001$ for all classes of follicles.

Class 1: $\leq 5 \mathrm{~mm}$, Class 2: 6 to $9 \mathrm{~mm}$ and Class 3: $\geq 10 \mathrm{~mm}$, based on the ultrasound machine caliper.

A pesar de que el semen utilizado provenía de machos de fertilidad comprobada, se evaluó el efecto del toro sobre las estructuras colectadas y se determinó que afectó el total de estructuras colectadas $(\mathrm{P}=0,018)$, el total de mórulas calidad $1(\mathrm{P}=0,047)$ y el total de embriones calidad $4(\mathrm{P}=0,036)$. Mientras que, el número de estructuras infertilizadas (ovocitos) y el total de embriones fertilizados, tendieron a diferir por efecto del toro ( $\mathrm{P}=0,087 \mathrm{y}$ $\mathrm{P}=0,085$, respectivamente).

\section{Relaciones entre las variables de respuesta}

Debido a que no se encontró un efecto de respuesta entre la suplementación con PPG y la actividad folicular durante el experimento, o cantidad y calidad de las estructuras colectadas, se exploraron relaciones entre las variables de respuesta del experimento. Se evaluó si la cantidad de folículos totales en el día de inicio del tratamiento de sincronización y multiovulación (día cero), así como la presencia de un cuerpo lúteo (ciclicidad), influyeron sobre el total de estructuras colectadas. Se encontró que ninguna de las dos variables (número de folículos o presencia de cuerpo lúteo) afectó el número de estructuras colectadas ( $\mathrm{P}=0,82$ y $\mathrm{P}=0,50$, respectivamente).

En el Cuadro 2 se muestra una matriz de correlaciones entre los resultados de las evaluaciones con ultrasonido del día 0 del experimento con los resultados de palpación y colecta de estructuras del día quince. Los folículos de clase 1 ( $\leq 5 \mathrm{~mm}$ de diámetro durante el día cero), fueron los que aportaron más al total de folículos encontrados en el día quince. 
Cuadro 1. Cantidad promedio de embriones transferibles (calidad 1 y 2) obtenidos en las diferentes repeticiones en cada tratamiento con (CPPG) y sin (SPPG) suplementación con propilenglicol en vacas razas Simbrah, Canchim (5/8 Charolais x 3/8 Nelore), Limousine y Nelore. Guápiles, Pococí, Costa Rica. Enero 2011 a febrero 2012.

Table 1. Average number of transferable embryos (quality 1 and 2) obtained in the different replicates in each treatment with (CPPG) and without (SPPG) propylene glycol supplementation in cows Simbrah, Canchim (5/8 Charolais x 3/8 Nellore), Limousine and Nellore breeds. Guapiles, Pococi, Costa Rica. January 2011 to February 2012.

\begin{tabular}{|c|c|c|c|c|c|c|c|}
\hline \multirow[t]{2}{*}{ Rep } & \multirow[t]{2}{*}{ Parámetro } & \multicolumn{2}{|c|}{ Calidad 1} & \multicolumn{2}{|c|}{ Calidad 2} & \multicolumn{2}{|c|}{ Total ET } \\
\hline & & SPPG & CPPG & SPPG & CPPG & SPPG & CPPG \\
\hline \multirow[t]{3}{*}{1} & Promedio* & $2,0 \pm 1,8$ & $10,0 \pm 9,6$ & $0,0 \pm 0,0$ & $2,0 \pm 2,6$ & 2,0 & 12,0 \\
\hline & Mínimo & 0,0 & 3,0 & 0,0 & 0,0 & $\mathrm{n}=4$ & $\mathrm{n}=3$ \\
\hline & Máximo & 4,0 & 21,0 & 0,0 & 5,0 & & \\
\hline \multirow[t]{3}{*}{2} & Promedio* & $11,0 \pm 13,9$ & $4,2 \pm 4,4$ & $0,0 \pm 0,0$ & $1,2 \pm 1,9$ & 11,0 & 5,5 \\
\hline & Mínimo & 2,0 & 0,0 & 0,0 & 0,0 & $\mathrm{n}=3$ & $\mathrm{n}=4$ \\
\hline & Máximo & 27,0 & 9,0 & 0,0 & 4,0 & & \\
\hline \multirow[t]{3}{*}{3} & Promedio* & $5,5 \pm 4,7$ & $5,7 \pm 4,0$ & $0,0 \pm 0,0$ & $0,0 \pm 0,0$ & 5,5 & 5,7 \\
\hline & Mínimo & 2,0 & 1,0 & 0,0 & 0,0 & $\mathrm{n}=4$ & $n=3$ \\
\hline & Máximo & 12,0 & 8,0 & 0,0 & 0,0 & & \\
\hline \multirow[t]{3}{*}{4} & Promedio* & $10,3 \pm 14,6$ & $4,7 \pm 4,0$ & $2,7 \pm 4,6$ & $0,0 \pm 0,0$ & 13,0 & 4,7 \\
\hline & Mínimo & 0,0 & 0,0 & 0,0 & 0,0 & $\mathrm{n}=3$ & $\mathrm{n}=3$ \\
\hline & Máximo & 27,0 & 7,0 & 8,0 & 0,0 & & \\
\hline \multirow[t]{3}{*}{5} & Promedio* & $7,3 \pm 2,1$ & $10,0 \pm 13,2$ & $0,7 \pm 1,2$ & $0,0 \pm 0,0$ & 8,0 & 10,0 \\
\hline & Mínimo & 5,0 & 0,0 & 0,0 & 0,0 & $\mathrm{n}=3$ & $n=3$ \\
\hline & Máximo & 9,0 & 25,0 & 2,0 & 0,0 & & \\
\hline \multirow[t]{3}{*}{6} & Promedio* & $11,0 \pm 15,6$ & $1,7 \pm 1,5$ & $0,5 \pm 0,7$ & $0,3 \pm 0,6$ & 11,5 & 2,0 \\
\hline & Mínimo & 0,0 & 0,0 & 0,0 & 0,0 & $\mathrm{n}=2$ & $n=3$ \\
\hline & Máximo & 22,0 & 3,0 & 1,0 & 1,0 & & \\
\hline \multirow{2}{*}{\multicolumn{2}{|c|}{$\begin{array}{l}\text { Total } \\
\text { Promedio }\end{array}$}} & & & & & 51 & 39,9 \\
\hline & & & & & & 8,5 & 6,65 \\
\hline
\end{tabular}

*Promedio \pm desviación estándar / *Average \pm standard deviation.

$\mathrm{Rep}=$ repetición, Total ET= total de embriones transferibles (calidad 1 y 2), n= número de vacas multiovuladas y colectadas en cada repetición / Rep= replicate, Total ET= total of transferable embryos (quality 1 and 2), n =number of multiovulated and collected cows per each replicate.

Se encontró que conforme aumentó el número de estructuras colectadas, el número de embriones totales y el número de embriones calidad 1 también aumentaron ( $\mathrm{r}=0,88$ y r=0,84, respectivamente), sin embargo, cuando aumentó el número de estructuras totales colectadas, también aumentó considerablemente el número de estructuras infertilizadas $(\mathrm{r}=0,78)$. La correlación más alta que se encontró fue entre el número de embriones totales y el número de embriones calidad 1 ( $\mathrm{r}=0,93)$.

\section{Discusión}

A pesar de que el PPG es un suplemento gluconeogénico (Chagas et al., 2007), y que los ovarios son glucodependientes (Peter et al., 2009), la suplementación con PPG no afectó la respuesta ovárica a los tratamientos multiovulatorios cuando se evaluó el número de folículos presentes por clase, por medio de ultrasonografía. Se observó una interacción entre el tratamiento y la repetición para los folículos clase 3, pero no se puede atribuir a 


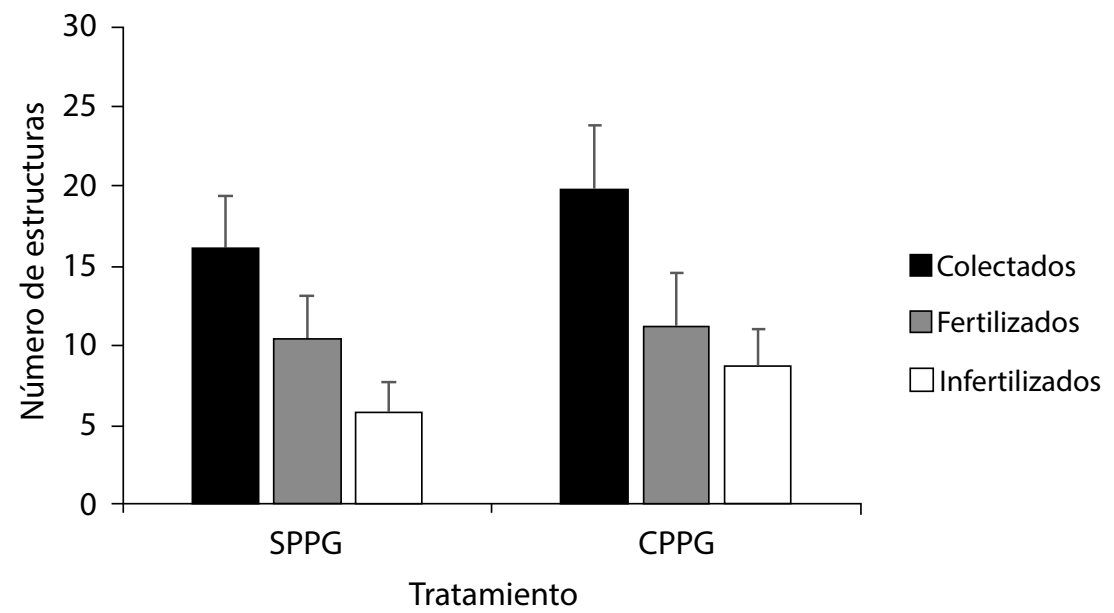

Figura 6. Cantidad promedio de estructuras colectadas por tratamiento con (CPPG) y sin (SPPG) suplementación con propilenglicol en vacas razas Simbrah, Canchim (5/8 Charolais x 3/8 Nelore), Limousine y Nelore. Guápiles, Pococí, Costa Rica. Enero 2011 a febrero 2012.

$\mathrm{P}>0,1$ para cada categoría de estructura colectada.

Colectados $=$ fertilizados+infertilizados, fertilizados $=$ embriones de diferentes calidades, Infertilizados $=$ ovocitos .

Figure 6. Average number of structures collected per treatment with (CPPG) and without (SPPG) propylene glycol supplementation in cows Simbrah, Canchim (5/8 Charolais x 3/8 Nellore), Limousine and Nellore breeds. Guapiles, Pococi, Costa Rica. January 2011 to February 2012.

$\mathrm{P}>0.1$ for each category of collected structure.

Collected $=$ fertilized + infertilized, Fertilized $=$ embryos of different qualities, Infertilized $=$ oocytes.

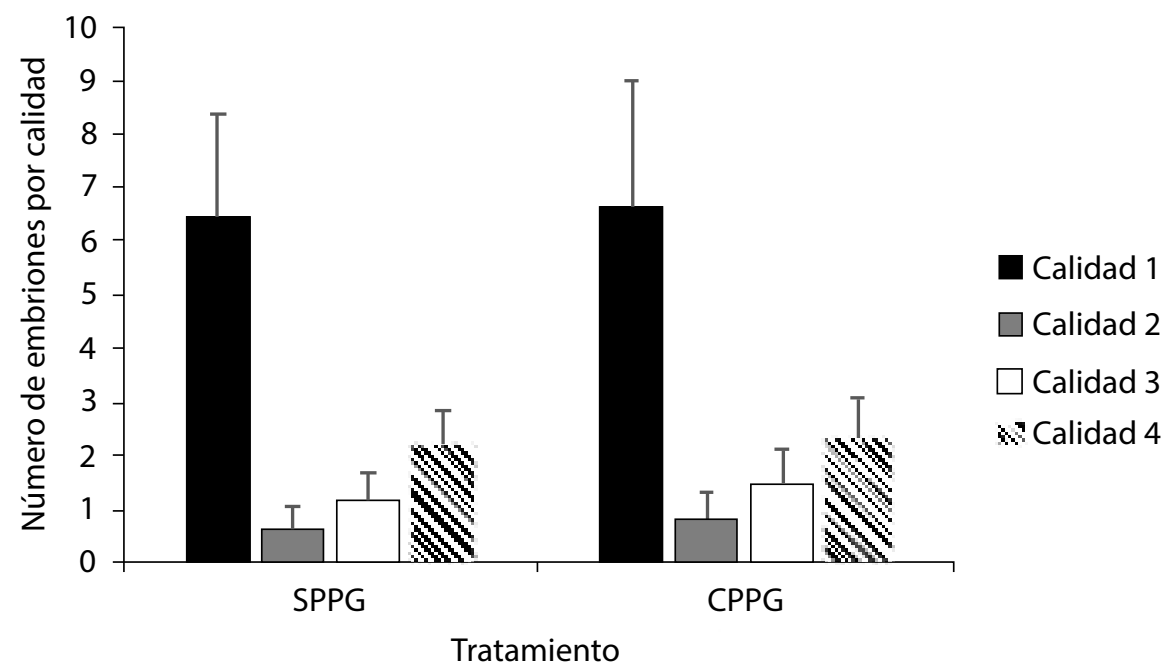

Figura 7. Distribución de las estructuras colectadas (embriones) según calidad por tratamiento con (CPPG) y sin (SPPG) suplementación con propilenglicol en vacas razas Simbrah, Canchim (5/8 Charolais x 3/8 Nelore), Limousine y Nelore. Guápiles, Pococí, Costa Rica. Enero 2011 a febrero 2012.

$\mathrm{P}>0,1$ para cada calidad según tratamiento.

Calidad de embriones definida según Bó y Mapletoft (2013).

Figure 7. Distribution of the collected structures (embryos) according to quality by treatment with (CPPG) and without (SPPG) propylene glycol supplementation in cows Simbrah, Canchim (5/8 Charolais x 3/8 Nellore), Limousine and Nellore breeds. Guapiles, Pococi, Costa Rica. January 2011 to February 2012.

$\mathrm{P}>0.1$ for each quality according to treatment.

Embryo quality defined according to Bó and Mapletoft (2013). 
Cuadro 2. Matriz de correlaciones entre variables cuantificadas en el experimento con (CPPG) y sin (SPPG) suplementación con propilenglicol en vacas razas Simbrah, Canchim (5/8 Charolais x 3/8 Nelore), Limousine y Nelore. Guápiles, Pococí, Costa Rica. Enero 2011 a febrero 2012.

Table 2. Correlation matrix among quantified variables in the experiment with (CPPG) and without (SPPG) propylene glycol supplementation in cows Simbrah, Canchim (5/8 Charolais x 3/8 Nellore), Limousine and Nellore breeds. Guapiles, Pococi, Costa Rica. January 2011 to February 2012.

\begin{tabular}{lccccccccccccc}
\hline & Foltot & Clase1 & Clase2 & CLUS & Clase3 & CLmano & Colect & Fert & Infert & Cal1 & Cal2 & Cal3 & Cal4 \\
\hline Foltot & 1 & 0,85 & 0,34 & 0,017 & 0,32 & 0,64 & 0,63 & 0,59 & 0,43 & 0,46 & 0,3 & 0,52 & 0,58 \\
Clase1 & & 1 & 0,1 & $-0,024$ & 0,24 & 0,6 & 0,62 & 0,65 & 0,35 & 0,45 & 0,41 & 0,65 & 0,69 \\
Clase2 & & & 1 & 0,14 & 0,18 & 0,38 & 0,37 & 0,29 & 0,31 & 0,26 & 0,2 & 0,031 & 0,35 \\
CLUS & & & & 1 & $-0,15$ & 0,12 & 0,08 & 0,047 & 0,092 & 0,13 & 0,11 & 0,07 & $-0,23$ \\
Clase3 & & & & & 1 & 0,25 & 0,13 & 0,59 & 0,43 & 0,46 & 0,31 & 0,52 & 0,58 \\
CLmano & & & & & 1 & 0,77 & 0,68 & 0,58 & 0,63 & 0,28 & 0,53 & 0,52 \\
Colect & & & & & & 1 & 0,88 & 0,78 & 0,84 & 0,33 & 0,58 & 0,69 \\
Fert & & & & & & & 1 & 0,38 & 0,93 & 0,58 & 0,66 & 0,76 \\
Infert & & & & & & & & 1 & 0,4 & $-0,13$ & 0,26 & 0,34 \\
Cal1 & & & & & & & & & 1 & 0,49 & 0,45 & 0,56 \\
Cal2 & & & & & & & & & & 1 & 0,28 & 0,29 \\
Cal3 & & & & & & & & & & & & 1 & 0,49 \\
Cal4 & & & & & & & & & & & & & \\
\hline
\end{tabular}

Foltot $=$ folículos totales al día 0, Clase $1=$ número folículos clase 1 ( $\leq 5 \mathrm{~mm}$ de diámetro $)$ al día 0 , Clase $2=$ número folículos clase 2 (5-9 mm de diámetro) al día 0 , Clase $3=$ número folículos clase 3 ( $\geq 10 \mathrm{~mm}$ de diámetro) al día 0, CLUS= cuerpo lúteo presente al día 0 (detectado por ultrasonido), CLmano= número de cuerpos lúteos en el día 15 (detectados por palpación), Colect= número de estructuras colectadas al día 15, Fert= número de embriones colectados al día 15, Infert= número de estructuras infertilizadas colectadas al día 15 , Cal1, Cal2, Cal3 y Cal4= clasificación del embrión de acuerdo a su calidad 1, 2, 3 o 4 / Foltot= total follicles at day 0, Clase1= follicle number class $1(\leq 5 \mathrm{~mm}$ diameter $)$ at day 0, Clase $2=$ follicle number class $2(5-9 \mathrm{~mm}$ diameter $)$ at day 0 , Clase $3=$ follicle number class 3 ( $\geq 10 \mathrm{~mm}$ diameter) at day $0, \mathrm{CLUS}=$ corpus luteum present at day 0 (detected by ultrasound), CLmano= number of corpora lutea at day 15 (detected by palpation), Colect= number of structures collected at day 15, Fert= number of embryos collected at day 15 , Infert= number of infertilized structures collected at day 15, Cal1, Cal2, Cal3 and Cal4= classification of the embryo according to its quality $1,2,3$ or 4 .

Los días que se especifican son relativos al experimento / The days specified are relative to the experiment.

los tratamientos, puesto que los datos fueron inconsistentes. Esto se debe probablemente a factores ambientales que afectaron la respuesta ovárica a través de las repeticiones.

Los resultados en cuanto al desarrollo folicular fueron variables a través del tiempo, incluso, en dos de las repeticiones del experimento, la cantidad de folículos clase 3 fue mayor en el grupo sin PPG, lo cual se opone a la hipótesis de este experimento, que esperaba mejorar la respuesta ovárica a través de la suplementación con PPG, sin embargo, resultados similares fueron reportados por Hackbart et al. (2017). El suministro de $250 \mathrm{ml}$ de PPG a hembras bovinas receptoras de embriones, mejoró en 20\% la implantación de los embriones, según Ordóñez et al. 2006, debido a una mejora en las estructuras ováricas, en específico a la mejora en la calidad del cuerpo lúteo (tamaño) y la producción de progesterona. Dicho efecto sobre las estructuras ováricas no se encontró en el presente estudio cuando los folículos de las hembras donantes fueron medidos y categorizados en clases, lo cual también coincide con Hackbart et al. (2017). Es probable que la alteración de la función folicular ovárica no se vea afectada por el PPG, por ejemplo, Butler et al. (2006) suplementaron $500 \mathrm{ml}$ de PPG a vacas de leche durante el periparto, y a pesar de que encontraron aumento en las concentraciones de insulina y glucosa en los animales suplementados, 
no se alteró el desarrollo folicular. Este aumento significativo en insulina y glucosa, sin un efecto positivo sobre el desarrollo folicular es confirmado por Hackbart et al. (2017).

$\mathrm{Al}$ analizar las estructuras colectadas, se encontró, independientemente del tratamiento, que la cantidad de embriones transferibles (calidad 1 y 2 según Bó y Mapletoft (2013)), fue mayor a los 6 - 7 embriones transferibles que reporta Hasler (2014). Esta diferencia, probablemente es debida a que las donantes utilizadas fueron seleccionadas como tales en trabajos previos.

La literatura disponible sobre suplementación con PPG en vacas o novillas donantes de embriones es escaza. En cabras donantes de embriones, Souza et al. (2008), suplementaron propilenglicol, pero a pesar de ser una especie distinta, tampoco encontraron que dicho suplemento mejorara la calidad de las estructuras colectadas, debido a que no se aumentó el número de embriones calidad 1 y 2. En el mismo estudio en cabras, además de la suplementación con propilenglicol, los autores evaluaron la inclusión de insulina como parte de uno de los tratamientos, en este caso, se obtuvo mejor calidad de embriones. Esto sugiere que no solamente es el efecto de una fuente gluconeogénica, sino el metabolismo completo de la glucosa el que favorece la reproducción. Por su parte, en vacas Hosltein, la suplementación con PPG redujo el total de ovulaciones y la tasa de fertilización (Hackbart et al., 2017).

A pesar de la inconsistencia de los resultados, el número de estructuras colectadas, fertilizadas (embriones) e infertilizadas (ovocitos), según el tratamiento, presentó diferencia numérica en cualquiera de las estructuras a favor del tratamiento con PPG.

La flexibilidad en los resultados de este experimento, hace suponer que la respuesta ante la multiovulación en vacas, se vio afectada por múltiples factores (Mikkola y Taponen, 2017; Perez et al., 2017), incluyendo el efecto paterno que fue significativo en este estudio. Esta multifactoriedad, dificulta la evaluación de la respuesta animal ante un suplemento.

Las evaluaciones por medio de ultrasonido, palpación, colecta y clasificación de embriones, permitieron observar relaciones entre variables, que influyen en la respuesta a la multiovulación. En ganado Bos indicus, se ha correlacionado positivamente el número de folículos presentes en el ovario con la respuesta a la multiovulación (Sartori y Barros, 2011). En este experimento, al evaluar la cantidad de folículos totales en el día de inicio del tratamiento de sincronización y multiovulación (día cero), así como la presencia de un cuerpo lúteo (ciclicidad) sobre el total de estructuras colectadas, se encontró que ninguna de las dos variables influyó sobre el número de estructuras colectadas, sin embargo, al evaluar únicamente los folículos de clase 1 ( $\leq 5 \mathrm{~mm}$ de diámetro durante el día cero), estos sí aportaron al total de folículos encontrados en el día quince, lo cual es congruente con que las hembras con más folículos responden mejor a los tratamientos de multiovulación (Sartori y Barros, 2011). Al hacer el análisis de correlaciones, se confirmó que a mayor población folicular en el día cero (inicio del tratamiento de sincronización-multiovualción) se obtiene mayor cantidad de ovulaciones (medido por la presencia de cuerpos lúteos) y se colectan más estructuras.

A nivel práctico, la ciclicidad de la donante es un requisito para la escogencia de la misma, pues se espera una mejor respuesta a la multiovulación de animales con actividad ovárica. Sin embargo, en este estudio, la presencia de un cuerpo lúteo al inicio del tratamiento de sincronización y multiovulación (día cero) de la donante, no afectó la eficiencia de la técnica, además, se encontró una asociación nula o muy baja con la cantidad y calidad de embriones en el día quince.

En general, la suplementación con PPG no afectó la cantidad y tamaño de estructuras ováricas medidas con ultrasonido en vacas donantes de embriones, ni la cantidad o calidad de embriones colectados. La respuesta a la multiovulación, al igual que la fertilidad en la hembra bovina, es afectada por múltiples factores, lo que aumenta la dificultad de encontrar resultados claros a partir de suplementos como el PPG. Factores ambientales como temperatura, precipitación, alimentación y factores intrínsecos al animal, pueden alterar la respuesta a la multiovulación (Ferraz et al., 2016). En este estudio, quizá una de las razones por las cuales no se obtuvo la respuesta esperada, es porque las donantes mantuvieron una adecuada nutrición base y condición corporal durante 
el experimento, esto limita la respuesta ante cualquier suplemento. El PPG se ha usado para mejorar la fertilidad de las vacas, sin embargo, la mejora se observa cuando se suministra en animales con condición corporal baja (Chagas et al., 2007). Otro factor capaz de minimizar la respuesta individual de las vacas, así como la respuesta ante un tratamiento, es el uso de protocolos de sincronización y estimulación folicular (Santos et al., 2009). Estos probablemente sobrepasan o mitigan otros efectos sobre la respuesta a la multiovulación y colecta de embriones.

\section{Conclusiones}

La suplementación con propilenglicol en vacas donantes de embriones no mejoró la cantidad y calidad de embriones transferibles.

Los tratamientos no estuvieron asociados con la respuesta en embriones transferibles, pero la cantidad obtenida fue mayor que la reportada en estudios previos.

Los resultados sugieren que otros factores afectan la respuesta ante la multiovulación, por lo que es necesario seguir investigando para maximizar la técnica de producción y transferencia de embriones in vivo, e incluir evaluaciones de hormonas metabólicas y reproductivas para contar con más indicadores ante la respuesta a los tratamientos.

\section{Agradecimientos}

Los autores desean expresar su agradecimiento a los señores Fabio Blanco y Guido Carballo Cruz, a los ganaderos que aportaron los animales a la Unidad de Reproducción Animal (URA), al personal de la URA, así como a la Vicerrectoría de Investigación y a la Fundación de la Universidad de Costa Rica por el apoyo brindado.

\section{Literatura citada}

ATSDR (Agency for Toxic Substances and Diseases Registry). 1997. Public health statement, proylene Glycol CAS\#:57-55-6. ATSDR, Atlanta, GA, USA.

Bó, G.A., and R.J. Mapletoft. 2013. Evaluation and classification of bovine embryos. Anim. Reprod. 10:344-348.

Butler, S.T. 2014. Nutritional management to optimize fertility of dairy cows in pasture-based systems. Animal 8:15-26. doi: $10.1017 /$ S 1751731114000834

Butler, S.T., S.H. Pelton, and W.R. Butler. 2006. Energy balance, metabolic status, and the first postpartum ovarian follicle wave in cows administered propylene glycol. J. Dairy Sci. 89:2938-2951. doi:10.3168/jds.S0022-0302(06)72566-8

Callesen, H., P. Løvendahl, A. Bak, and T. Greve. 1995. Factors affecting the developmental stage of embryos recovered on day 7 from superovulated dairy cattle. J. Anim. Sci. 73:1539-1543. doi:10.2527/1995.7361539x

Chagas, L.M., P.J. Gore, S. Meier, K.A. Macdonald, and G.A. Verkerk. 2007. Effect of monopropylene glycol on luteinizing hormone, metabolites, and postpartum anovulatory intervals in primiparous dairy cows. J. Dairy Sci. 90:1168-1175. doi:10.3168/jds.S0022-0302(07)71603-X

Chebel, R.C., D.G. Demétrio, and J. Metzger. 2008. Factors affecting success of embryo collection and transfer in large dairy herds. Theriogenology 69:98-106. doi:10.1016/j.theriogenology.2007.09.008

Christensen, J.O., R.R. Grummer, F.E. Rasmussen, and S.J. Bertics. 1997. Effect of method of delivery of propylene glycol on plasma metabolites of feed-restricted cattle. J. Dairy Sci. 80:563-568. doi:10.3168/jds.S0022-0302(97)75971-X 
Chung, Y.H., C.M. Martinez, N.E. Brown, T.W. Cassidy, and G.A. Varga. 2009. Ruminal and blood responses to propylene glycol during frequent feeding. J. Dairy Sci. 92:4555-4564. doi:10.3168/jds.2009-2131

Dijkstra, J. 1994. Production and absorption of volatile fatty acids in the rumen. Livest. Prod. Sci. 39:61-69. doi:10.1016/03016226(94)90154-6

Ferraz, P.A., C. Burnley, J. Karanja, A. Viera-Neto, J.E.P. Santos, R.C. Chebel, and K.N. Galvão. 2016. Factors affecting the success of a large embryo transfer program in Holstein cattle in a commercial herd in the southeast region of the United States. Theriogenology 86:1834-1841. doi:10.1016/j.theriogenology.2016.05.032

Hackbart, K.S., R.W. Bender, P.D. Carvalho, L.M. Vieira, A.R. Dresch, J.N. Guenther, H. Gencoglu, A.B. Nascimento, R.D. Shaver, and M.C. Wiltbank. 2017. Effects of propylene glycol or elevated luteinizing hormone during follicle development on ovulation, fertilization, and early embryo development. Biol. Reprod. 97:550-563. doi:10.1093/biolre/iox050

Hasler, J.F. 2014. Forty years of embryo transfer in cattle: A review focusing on the journal Theriogenology, the growth of the industry in North America, and personal reminisces. Theriogenology 81:152-169. doi:10.1016/j. theriogenology.2013.09.010

Hoedemaker, M., D. Prange, H. Zerbe, J. Frank, A. Daxenberger, and H.H. Meyer. 2004. Peripartal propylene glycol supplementation and metabolism, animal health, fertility, and production in dairy cows. J. Dairy Sci. 87:2136-2145. doi:10.3168/jds.S0022-0302(04)70033-8

Jenkins, N.T., G. Peña, C. Risco, C.C. Barbosa, A. Vieira-Neto, and K.N. Galvão. 2015. Utility of inline milk fat and protein ratio to diagnose subclinical ketosis and to assign propylene glycol treatment in lactating dairy cows. Can. Vet. J. 56 850-854.

Laskowski, D., Y. Sjunnesson, P. Humblot, G. Andersson, H. Gustafsson, and R. Båge. 2016. The functional role of insulin in fertility and embryonic development-What can we learn from the bovine model? Theriogenology 86:457-464. doi:10.1016/j.theriogenology.2016.04.062

Mikkola, M., and J. Taponen. 2017. Embryo yield in dairy cattle after superovulation with Folltropin or Pluset. Theriogenology 88:84-88. doi:10.1016/j.theriogenology.2016.09.052

Moallem, U., M. Katz, H. Lehrer, L. Livshitz, and S. Yakoby. 2007. Role of peripartum dietary propylene glycol or protected fats on metabolism and early postpartum ovarian follicles. J. Dairy Sci. 90:1243-1254. doi 10.3168/jds.S00220302(07)71613-2

Ordóñez, O.H., C.T. Miguel, E.G. Piñeiro, N.F. Fernandez, y C.D. Manforte. 2006. El propilenglicol mejora los resultados de la transferencia de embriones. Sito Argentino de Producción Animal, ARG. http://www.produccionbovina.com.ar/ informacion_tecnica/transplante_embrionario/20-propilenglicol.pdf (consultado 8 feb. 2017).

Palma, G.A. 2008. Biotecnología de la reproducción. ReproBiotec, Mar del Plata, ARG.

Perez, B.C., J.C. Balieiro, R.V. Ventura, F. Bruneli, and M.G. Peixoto. 2017. Inbreeding effects on in vitro embryo production traits in Guzerá cattle. Animal 17:1-8. doi:10.1017/S1751731117000854

Peter, A.T., L.A. Vos, and D.J. Ambrose. 2009. Postpartum anestrus in dairy cattle. Theriogenology 71:1333-1342. doi:10.1016/j. theriogenology.2008.11.012

Piantoni, P., and M.S. Allen. 2015. Evaluation of propylene glycol and glycerol infusions as treatments for ketosis in dairy cows. J. Dairy Sci. 98:5429-5439. doi:10.3168/jds.2015-9476

Santos, J.E. 2008. Impact of nutrition on dairy cattle reproduction. In: High Plains Dairy Conference, editor, Proceedings of the High Plains Dairy Conference. High Plains Dairy Conference, Amarillo, TX, USA. p. 25-36. 
Santos, J.E., H.M. Rutigliano, and M.F. Sá-Filho. 2009. Risk factors for resumption of postpartum estrous cycles and embryonic survival in lactating dairy cows. Anim. Reprod. Sci. 110:207-221. doi:10.1016/j.anireprosci.2008.01.014

Sartori, R., and C.M. Barros. 2011. Reproductive cycles in Bos indicus cattle. Anim. Reprod. Sci. 124:244-250. doi:10.1016/j. anireprosci.2011.02.006

Souza, A.L., G. Galeati, A.P. Almeida, I.J. Arruda, N. Govoni, V.J. Freitas, and D. Rondina. 2008. Embryo production in superovulated goats treated with insulin before or after mating or by continuous propylene glycol supplementation. Reprod. Domest. Anim. 43:218-221. doi:10.1111/j.1439-0531.2007.00881.x

Windholz, M., S. Budavari, and M. Co. 1983. The Merck index : an encyclopedia of chemicals, drugs, and biologicals. NCBI, MD, USA. https://www.ncbi.nlm.nih.gov/nlmcatalog/8405389 (accessed 8 Feb. 2017). 